\title{
Mechanisms Underlying Non-Pharmacological Dementia Prevention Strategies: A Translational Perspective
}

\author{
V. Alanko ${ }^{1,2}$, C. Udeh-Momoh ${ }^{1,3}$, M. Kivipelto ${ }^{1,3,4,5}$, A. Sandebring-Matton ${ }^{1,2,3, \#}$
}

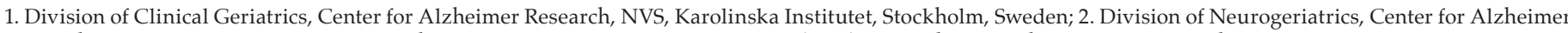

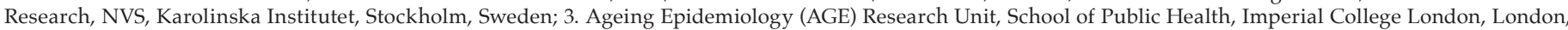

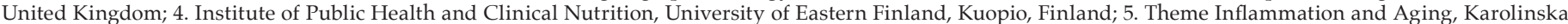
University Hospital, Solna, Sweden

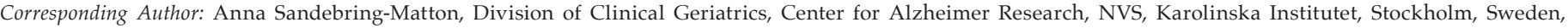
anna.matton@ki.se, telephone +46-8-524 800 00, fax +46-8-31 1101

\begin{abstract}
Since developing an effective treatment for Alzheimer's disease (AD) has been encountered as a challenging task, attempts to prevent cognitive decline by lifestyle modifications have become increasingly appealing. Physical exercise, healthy diet, and cognitive training are all modifiable, non-pharmacological lifestyle factors considered to influence cognitive health. Implementing lifestyle modifications on animal models of AD and cognitive impairment may reveal underlying mechanisms of action by which healthy lifestyle contribute to brain health. In mice, different types of lifestyle interventions have been shown to improve cognitive abilities, alleviate AD-related pathology and neuroinflammation, restore mitochondrial function, and have a positive impact on neurogenesis and cell survival. Different proteins and pathways have been identified to mediate some of the responses, amongst them BDNF, Akt-GSK3 $\beta$, JNK, and ROCK pathway. Although some important pathways have been identified as mediating improvements in brain health, more research is needed to confirm these mechanisms of action and to improve the understanding of their interplay. Moreover, multidomain lifestyle interventions targeting multiple risk factors simultaneously may be a promising avenue in future dementia prevention strategies. Therefore, future work is needed to better understand the synergistic impact of combinatory lifestyle strategies on cellular mechanisms and brain health.
\end{abstract}

Key words: Alzheimer's disease, dementia, prevention, mouse models, multidomain, lifestyle.

\section{Introduction}

7 he high global prevalence of the leading neurodegenerative disease-causing dementia, Alzheimer's disease (AD) has been linked to exponential increases in ageing populations worldwide (1), though trends of attenuated incidence in highincome countries have been observed (2). Due to the slow progress in $\mathrm{AD}$-dementia drug development, dementia prevention has become increasingly important and has drawn significant attention during the past few decades. Recent reports have been disseminated in the last few Received October 26, 2021

Accepted for publication December 9, 2021 years underpinning the possibilities and importance of considering lifestyle factors for dementia prevention (3). Livingston et al.'s seminal report (2020) suggested that as much as $40 \%$ of dementia risk is attributable to lifestyle factors (4). Around the same time, the World Health Organization published guidelines for lifestyle modification to reduce the risk of cognitive decline and dementia (5).

Dementia prevention has been investigated both by observational studies and randomized controlled trials (RCT), both with their respective benefits and limitations (6). Still, there is an incomplete understanding of the underlying mechanisms of action. Although brain changes and functions can be assessed during preventive interventions using neuroimaging techniques and fluid biomarkers, such methods will not reveal the complete mechanisms underlying the alterations. Identifying these mechanisms could result in new treatment strategies. To better understand probable underlying mechanisms, it is necessary to conduct prevention studies in pre-clinical AD models.

While several modifiable risk factors have been identified, in this review we will discuss the effects of lifestyle interventions rather than pharmacological strategies that may directly impact risk factors such as hypertension and diabetes. We consider physical exercise, diet, and cognitive training as the main preventive factors, as these may either directly or indirectly impact some of the other risk factors, including hypertension, obesity, and diabetes (7-9). We will further describe current evidence from non-pharmacological pre-clinical prevention studies in mouse models of mainly AD and ageing, and how they may be translated to clinical applications. 


\section{Physical exercise}

\section{AD-pathology, inflammation, and glial alterations}

Extensive studies performed on dementia mouse models exposed to exercise training report positive outcomes in cognitive tests (10-18). Many of these studies report an increase of synaptic markers indicating preservation of synapses and their function in the brains of exercising mice $(10-14,19)$. Additionally, desired alterations in AD-related pathology after a period of exercise in AD transgenic mice have been observed in several studies $(10-12,15,20)$. Physical training can induce the phosphorylated Akt/phosphorylated GSK3 $\beta$ system, which in turn may reduce tau pathology $(11,14)$. Exercise attenuates pro-inflammatory markers, microglia and astrocyte activation, and increases anti-inflammatory markers in mice; such changes have been observed in $3 \times \mathrm{Tg}-\mathrm{AD}$ mice as a result of different exercise paradigms $(11,12,21)$. In APP/PS1 mice, exercise reduced the count and intensity of plaque-associated astrocytes (20) while in physically active 5xFAD mice they were increased (13). The reduced neuroinflammation is suggested to be mediated via inhibition of the c-Jun N-terminal kinase (JNK) pathway (11) or activation of the microRNA miR129-5p (16).

White matter is also affected early in $\mathrm{AD}$ progression (22). Total white matter volume decreases in mouse models of $\mathrm{AD}$ (17) but can be restored to the levels of wild type (WT) mice (17) or significantly increased compared to sedentary counterparts (23) with exercise. Decreased myelin in old WT mice can be improved by exercise (24), yet with conflicting results (25). Indeed, exercise seems to promote differentiation of oligodendrocyte progenitor cells and increase the number of mature oligodendrocytes in mice (26). Axon growth can be inhibited by myelinassociated factors via RhoA / Rho kinase (ROCK) pathway $(24,27)$, but also differentiation of oligodendrocytes is inhibited by the same pathway (28). Physical activity downregulates the ROCK pathway, which is suggested to mediate the increased myelination. Exercise further improves vasculature in the brain, particularly in the white matter, which otherwise is compromised in APP/ PS1 mice (17).

\section{Neurogenesis $\mathcal{E}$ anti-apoptotic pathways}

One common finding in several pre-clinical studies is the increased levels of the brain-derived neurotrophic factor (BDNF) as a result of physical activity $(10,13$, $14,19,29-31)$. BDNF expression is regulated to a great extent by other muscle-derived myokines, such as FNDC5 (fibronectin type III domain containing 5) and its cleavage product Irisin (32). FNDC5 expression is regulated by peroxisome proliferator-activated receptor gamma coactivator 1-alpha (PGC-1 $\alpha$ ) (32). Expression of the mitochondrial biogenesis regulator PGC- $1 \alpha$ may be induced by exercise $(11,12)$, although conflicting results have been reported (21). Furthermore, BDNF has a precursor form (proBDNF) and a mature form (hereafter referred to as BDNF), where proBDNF binds the receptor p75 and its co-receptor, Sortilin, activating for instance apoptotic signalling while BDNF mediates its effects via tropomyosin-related kinase B (TrkB) receptor signalling (33). Extracellular cleavage of proBDNF is executed by several different proteases into the mature form (34). There are at least two upstream regulators of BDNF processing, tissue-type plasminogen activator (tPA) and urokinase-type plasminogen activator (uPA), which process plasminogen to plasmin that in turn cleaves proBDNF to $\operatorname{BDNF}(35,36)$. Interestingly, tPA is activated by physical exercise (36). In the hippocampus, BDNF is a facilitator of long-term potentiation (LTP) and thus enhances memory and learning, neurogenesis, and synaptic plasticity. FNDC5/Irisin is involved in maintaining synaptic plasticity by stimulating BDNF expression, and levels of these proteins are reduced in the AD brain and CSF (18). When these are downregulated, LTP is impaired. The levels can, however, be restored by exercising, and thus memory can be improved or maintained (18). Additionally, exercise activates other neurotrophic factors involved in neurogenesis, as Kim and colleagues (12) did observe an increase in TrkB expression while BDNF levels were unchanged.

In 3xTg-AD mice, physical activity restored the levels of BrdU/NeuN-positive cells to the WT levels (12) or at least significantly increased compared to sedentary controls (14). In 5xFAD mice such effects have not been reported (13). An increase in newly formed neuronal precursors have also been shown in active WT mice $(25,37)$. Horowitz et al. (30) showed that not only was neurogenesis increased in exercising WT mice, but even in inactive aged mice when administered with plasma from the exercised mice. Similar results were reported when 3xTg-AD mice were administered plasma from young, exercised mice (19). This denotes that there is a clear axis between the training-induced systemic changes and the brain. Horowitz et al.'s study (30) specifically investigated the role of Gpld1 (glycosylphosphatidylinositol-specific phospholipase D1) and connected it to the increased BDNF levels and neurogenesis. The liver produces increased levels of Gpld1 in exercised mice, but also in physically active persons (30). However, the compound mainly remains in the periphery (30). Reflecting on the report, the effect on BDNF could be attributed to a Gpld1uPA-plasminogen cascade. Finally, these effects were observed as an improved cognitive performance of the mice $(19,30)$.

Complementary to neurogenesis, exercise might also alleviate cell death in the brain $(14,15,19,24)$. This effect has been attributed to a decrease in pro-apoptotic markers like caspases, cytochrome c, and Bax, and additionally 
to an increase in anti-apoptotic markers like Bcl-2 (14, $15,24,31)$. Downregulation of the ROCK system is a plausible pathway reducing apoptosis (24), and as a downstream target of receptor p75 (38) the effect could be attributed either to a reduction in proBDNF or increase in BDNF. In exercised $\mathrm{AD}$ mice $(14,15)$ or in $\mathrm{AD}$ mice administered plasma from exercised mice (19) the levels of these different proteins are not necessarily restored to a WT level, but still significantly improved compared to sedentary mice. Intriguingly, resistance training did not affect Bax and Bcl-2 levels (11).

\section{Metabolic factors $\mathcal{E}$ mitochondrial function}

Glucose hypometabolism is a known feature of $\mathrm{AD}$ (39) and many mouse models with memory impairment demonstrate a deficiency in glucose uptake $(10,40)$. Exercise can induce an increase in brain expression of glucose transporters GLUT1 and GLUT3 in APP/PS1 double-transgenic mice (10) as well as GLUT1 in NSE/ APPswe mice (31). In addition to improved brain glucose homeostasis, physical activity improves peripheral glucose metabolism in 3xTg-AD mice (21). In accordance with improved glucose metabolism, exercise alleviated insulin resistance in high-fat-diet fed WT mice by activating insulin receptors substrate (IRS) and its downstream pathways PI3K-PDK-1-Akt-GSK3 $\beta$ (37).

An additional effect that physical activity appears to have on the brain is maintenance of mitochondrial function. Kim and colleagues (12) detected restoration of mitochondrial length and enhanced mitochondrial biogenesis in exercised AD mice. Likewise, in APP/ PS1 mice, exercise recovered mitochondrial integrity and partly the ATP levels (10). When 3xTg-AD mice were administered with plasma from young, exercised mice, it resulted in a better capacity to maintain calcium homeostasis and in reduction of reactive oxygen species when compared to the other AD mice - yet the mitochondrial function was not returned to the WT levels (19). A similar effect was evident in 3xTg-AD mice undergoing treadmill exercise combined with light flickering (14). One possible mechanism of these effects could be the restoration of brain iron dyshomeostasis that contributes to oxidative stress (15).

\section{Nutrition}

\section{Olive oils and polyphenols}

There have been several attempts mimicking the Mediterranean diet (MeDi) in animal studies. As composing a full MeDi for mice is largely impossible, studies have investigated the effects of single nutrients or groups of nutrients that are thought to be the main health-promoting components of MeDi. Many of these nutrients are found in olive products and fish oils. Extra virgin olive oil-enriched diet improved working and spatial memory and increased synaptophysin expression in 3xTg-AD mice (41). Furthermore, both $A \beta$ and tau pathology were diminished in the treatment group, active microglia alleviated, and autophagy seemed to be induced (41). In the APP mutant TgSwDI mice, A $\beta$ pathology was ameliorated and some phosphorylated tau species were reduced by extra virgin olive oil consumption (42). The reduction of $A \beta$ plaques and vascular depositions of $A \beta$ was coupled to an increased clearance of the protein but even to a favourable APP processing pattern (42). Improved clearance was to some extent a result of increased expression of transporter proteins ApoE and ABCA1 (Apolipoprotein E and ATPbinding cassette transporter, respectively) (42).

Moreover, many studies have investigated the role of individual bioactive components of olive oil. Particular interest has been in studying the relationship between polyphenols found in olive oil and mitochondrial dysfunction and oxidative stress since polyphenols possess antioxidant activities (43). In APP/PS1 mice, hydroxytyrosol diet did not cause any major cognitive improvement nor did it affect $A \beta$ pathology (44). Nonetheless, hydroxytyrosol reversed oxidative stress in the brains and restored mitochondrial protein levels, reduced the levels of the cleaved caspase 3 , and alleviated neuroinflammation through inhibition of JNK pathway (44). Impacts on mitochondria have further been studied in aged NMRI mice fed with oleocanthal or ligstroside (45). Neither of the supplements affected the cognitive abilities of the mice, though those were not extensively studied. However, the life expectancy and cerebral ATP levels improved due to ligstroside diet, and a rationale that olive polyphenols improve mitochondrial respiration is supported by in vitro studies (45). In NMRI mice, a cocktail of purified secoiridoid polyphenols, including for example hydroxytyrosol, elevated ATP levels and moreover restored cognitive abilities (46). With oleuropein aglycone diet the AD mouse model TgCRND8 improved cognitive performance $(47)$ as well as reduced A $\beta$ load in different brain areas $(47,48)$. The diet further induced autophagy and histone acetylation $(47,48)$.

\section{Fish oils and Fortasyn Connect}

Fortasyn Connect (FC) is a multi-nutrient supplement consisting of docosahexaenoic acid (DHA), eicosapentaenoic acid (EPA), uridine monophosphate, choline, vitamins B12, B6, C, E, and folic acid, phospholipids, and selenium (49). FC diet has been shown to partly reverse cognitive deficits in APP/PS1 mice $(50,51)$. Yet, in another study with the same mouse model and diet, no such findings were reported (52), even though all three research settings have applied Morris Water Maze (MWM) tasks as the testing paradigm. ApoE4 and ApoE knock-out (KO) mice - models featuring the main genetic risk factor of sporadic AD - did not either improve learning nor memory in this particular cognitive 
test after FC diet (53). Although APP/PS1 mice improved learning after ingesting $\mathrm{FC}$, the dietary impacts on $\mathrm{A} \beta$ burden, inflammation, and reactive oxygen species were very minor overall, as were the effects with fish oils or a combination of fish oils and plant sterols (50). However, when the dietary intervention was initiated at an earlier time-point (compare 3 months of age (54) versus 5 months of age (50)), even if the intervention was of shorter duration, the $A \beta$ pathology was profoundly alleviated in the APP/PS1 mice (54). These results highlight the importance of early nutritional intervention for an impact on $A \beta$ neuropathology. Moreover, while the FC diet seems to have a positive effect on the $A \beta$ burden at an early stage of disease progression, this was not the case for a diet only enriched with DHA and uridine monophosphate, nor with either of them used alone (54).

Intriguingly, Jansen et al. (52) observed a significant increase in doublecortin-positive cells with the FC diet in APP/PS1 mice, indicating that the FC enhances neurogenesis. The level of these cells was restored to the WT level. On the contrary, a diet enriched only with DHA, EPA, and uridine monophosphate did not affect the number of doublecortin-positive cells (52), and the FC diet did not significantly increase the number of these cells in ApoE4 or ApoE KO mice (53). In APP/PS1 mice, the FC diet had also a positive impact on the degenerative burden of the cortex (54). Both of the diets did still result in a change in brain fatty acid composition, for example, by increasing the omega- 3 to omega- 6 ratio significantly and altering levels of brain oxysterols (52-54).

\section{Environmental enrichment and cognitive training}

\section{Cognitive training}

Cognitive training per se and its effects on cognitive abilities has not been extensively studied in mice particularly in settings where the retention time is long, i.e. the time between training and testing. Nevertheless, the lack of research with long retention time was addressed in a recent study investigated APP/PS1 mice in different cognitive training set-ups (55). In the first setting (referred to as "trained") mice were trained with MWM at the age of three months, and in the second setting ("overtrained") at the age of two months and again at three months. Importantly, training was scheduled before or at the time of plaque formation. However, the study did not include control groups that would not receive training. In both set-ups, mice were tested at age of seven months with a similar setting as during the training periods, but with an additional six-day-long reversal task. At seven months, "trained" transgenic mice showed rescued memory, although there was a significant group difference compared to WT littermates that underwent the same training program. Such difference was not observed between the groups of "overtrained" mice. In further analyses, "overtrained" APP/PS1 mice performed overall at the same level as WT mice and better compared to "trained" APP/PS1 mice. No molecular nor cellular changes were assessed in the study.

In an earlier study, Tg2576 mice were trained with MWM (56). The researchers included non-training control groups, both transgenic and WT littermates. The day after the water maze tasks, mice were subjected to Contextual Fear Conditioning where trained mice performed significantly better than non-trained transgenic mice. Furthermore, training enhanced LTP and restored dendritic complexity in the hippocampus to a WT level. Training also increased levels of some postsynaptic proteins - an effect that was coupled to activation of calcium/calmodulin-dependent protein kinase II (CaMKII) - and ameliorated AD-related pathology.

A large-scale study by Billings et al. (57), investigated the effects of water maze training throughout the lifetime of 3xTg-AD mice, with training sessions once every three months beginning at the age of two months. Until approximately the age of 12 months, transgenic mice had a clear benefit of training and were performing at a WT level, yet at 15 months of age, this effect started to diminish. In addition to the learning and memory improvements, the harmful $A \beta$ load was attenuated in 12 -month-old trained mice, and tau phosphorylation was reduced possibly through reduced activity of GSK3 $\beta$. Still, the favourable effects of cognitive training were most prominent when the training began before the emergence of neuropathology, although a later start of the training paradigm also improved the mice's abilities. On the contrary, in another study, even if 16-monthold PDAPP mice demonstrated faster forgetting than WT counterparts already seven days post-training, their memory could be retrieved with a short retraining period at seven weeks after the initial training period (58).

MWM is, however, stressful for mice. Although the improved memory in both Tg2576 (56) and 3xTg-AD (57) mice can be attributed to the cognitive training, as the control groups exposed to swimming only did not perform on the same level during cognitive testing, stress is an important component affecting physiology and learning. Certain amount of stress enhances learning, yet highly stressed individuals may encounter an opposing effect (59). Future research should aim to implement less stressful cognitive training paradigms to measure a more accurate effect on cognitive performance.

\section{Environmental enrichment}

An alternative to cognitive training is utilising environmental enrichment as a cognitive stimulator in pre-clinical research (60). Environmental enrichment can compose of different factors and components, but generally, it includes larger cages compared to standard housing together with various objects, such as tunnels, ladders, nesting material, and items of different sizes 
Figure 1. Summary figure of intervention effects

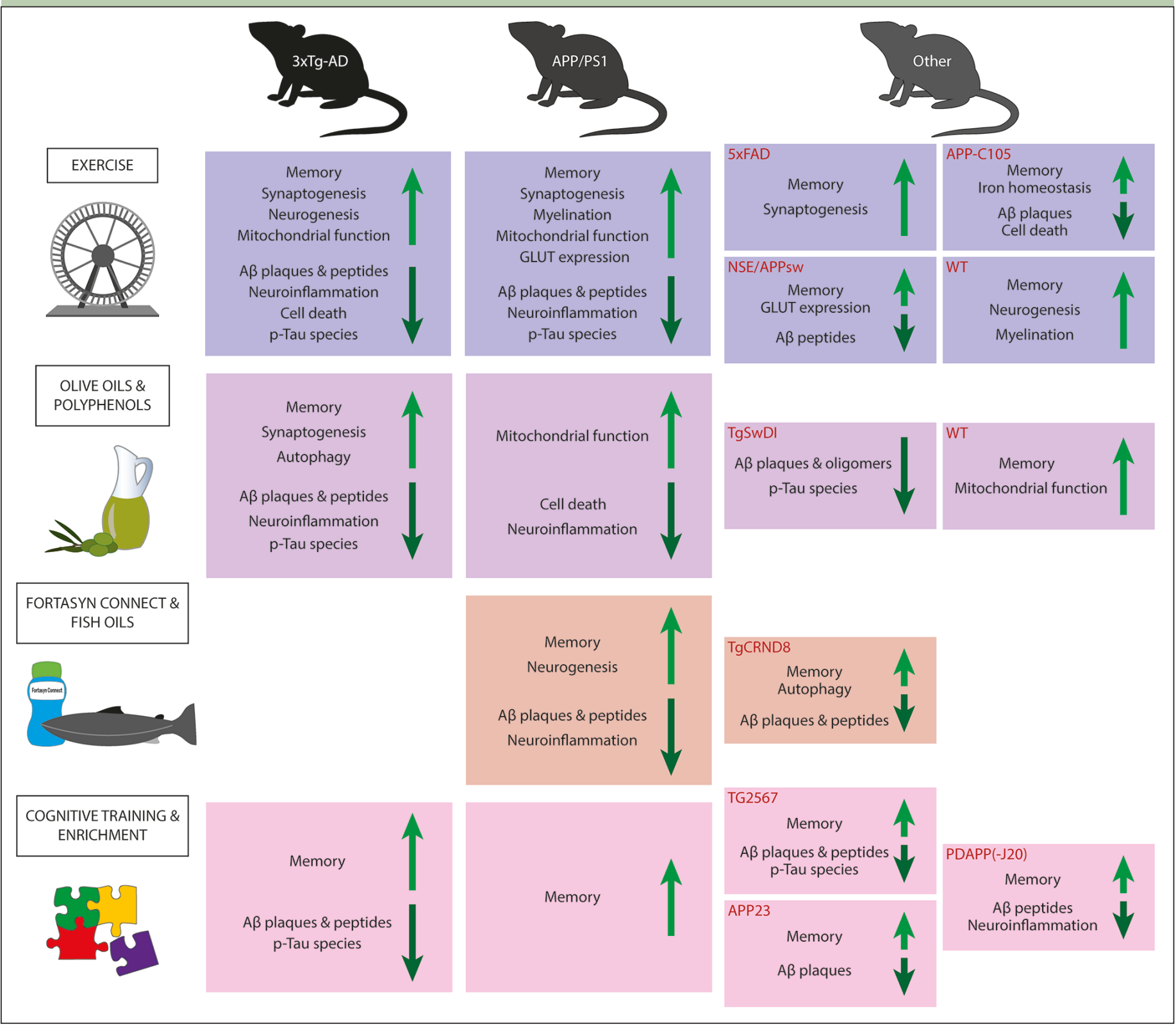

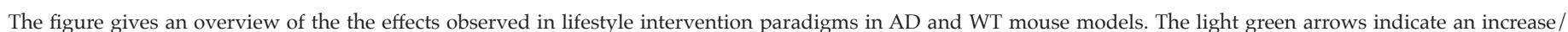

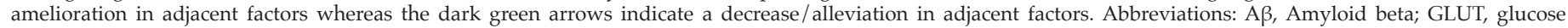
transporter; p-Tau, phosphorylated tau; WT, wild type.

and colours (60). These all contribute not only to enhanced cognitive stimulus but even to sensory and motor stimulations (60). Moreover, cages may also have running wheels and house a greater number of animals simultaneously. For this review, we want to separate the impacts of environmental enrichment and exercise, and therefore only studies implementing environmental enrichment paradigms excluding running wheels are discussed.

Independent of the time point of intervention start, enriched environment restored recognition memory in Tg2576 mice (61). However, spatial memory, as measured by MWM, was rescued only in those mice that lived in the enriched environment before the emergence of neuropathology. Enriched housing additionally counteracted the build-up of $A \beta$ pathology in these mice. APP23 mice housed in an enriched environment have demonstrated improvements both in MWM and Novel Object Recognition (62). Moreover, environmental enrichment induced both hippocampal and cortical BDNF expression and reduction in $A \beta$ plaque formation (62). On the contrary, in PDAPP-J20 mice that underwent enrichment intervention, a significant reduction in $A \beta 1-40$ and $A \beta 1-42$ peptides were observed, while the plaque load was unchanged (63). The volume of plaque-associated GFAP-positive astrocytes was also 
unaltered. Still, the volume of non-plaque-associated GFAP-positive cells was restored to a WT level due to environmental enrichment in these mice that otherwise have a significantly reduced number of astrocytes. Furthermore, the non-plaque-associated astrocytes in mice from enriched housing did not have as complex morphology as in standard housed mice.

Additionally, effects between exercise and environmental enrichment have been compared (64). In APP23 transgenic mice that were housed either in enriched cages or in a standard cages equipped with running wheels no effects on $A \beta$ load were observed (64). Still, the mice living in an enriched environment demonstrated improved learning. Based on analysis of BrdU- and doublecortin-positive cells, hippocampal neurogenesis was not induced in either intervention group. However, a marker for a postmitotic phase, Calretinin, was increased in mice living in enriched cages. Levels of neurotrophin and BDNF expression were also increased in these mice compared to controls while such effects were not evident in the exercised mice.

\section{Discussion \& future directions}

There is a high demand for strategies to cure and prevent dementia. Several of the herein discussed preclinical studies have reported clear benefits induced by singular lifestyle domain in mice (summarised in Fig. 1). When studying the effects in humans, some clinical trials investigating the effects from single-domain lifestyle changes have reported positive results, but likewise nonefficacious studies have been published (reviewed in 65).

Contradictions in efficacy between clinical and preclinical lifestyle intervention studies may be explainable by various considerations. First and foremost, mice are not men, and although there is great homology between brains, the significant inter-species differences in, for instance, cell types (66) may direct how the brain is influenced by various interventions. The issue is similar regarding biological homogeneity between mice with the same genetic background. In mice, it is near impossible to replicate the huge heterogeneity of different genetic and environmental risk factors present in humans. Considering $\mathrm{AD}$, the transgenic mouse models developed for $\mathrm{AD}$ research replicate mainly familial $\mathrm{AD}$ instead of the more common sporadic AD. Thus, it is dubious to translate findings from these mice to sporadic AD. In attempts to translate findings, the great majority of drugs developed for AD have failed to reach their primary clinical outcomes (67). Additionally, mice lack the variation in living conditions experienced by humans. This results in mice adhering to the intervention naturally more stringently than humans. A factor that however remains to be explored is how the synergy of different lifestyle interventions affects the brain on a cellular and molecular level. Such questions could be investigated in mice exposed to multimodal lifestyle intervention strategies even if the discrepancies between mice and men are acknowledged.

Exercise seems to be the most studied lifestyle factor regarding molecular and cellular mechanisms in mice. Increased BDNF levels are likely to be one of the primary mediating players of different positive outcomes when exercising. BDNF impacts several downstream pathways, for example, reducing levels of Bax and boosting expression of Bcl-2 (as in (14)), thus promoting cell survival, inducing expression of glucose transporters (as in (10)), activating Akt for phosphorylation of GSK3 $\beta$ (68), hence suppressing tau phosphorylation, and mediating neurogenesis $(30,37)$. Physical training promotes the expression of PGC- $1 \alpha(11,12)$ and FNDC5/ irisin (18) in the brain; factors that further contribute to the upregulation of BDNF expression. After translation, proBDNF can be further processed into mature BDNF. tPA levels increase due to exercise and hence increase the $\mathrm{BDNF}$ / proBDNF ratio (36). The exercise-induced BDNF levels could likely be attributed also to elevated liverderived Gpld1 levels that in turn promote plasminogen processing through uPA (30). BDNF levels were mainly measured in exercise-related studies, but also a couple of studies report elevated brain BDNF levels as a result of environmental enrichment $(62,64)$.

GSK3 $\beta$ is considered to have a significant role in AD pathogenesis (69) and has been studied in several of the pre-clinical studies discussed in this review. Cognitive training (57) and physical exercise $(11,14,37)$ have been found to increase the inactive, phosphorylated form of GSK3 $\beta$ and/or reduce the levels of the active form. These alterations have been accompanied by reductions in levels of its substrate phospho-tau $(11,14,57)$. Nonetheless, even if infusion of plasma from young, exercised mice into AD mice had ameliorated several pathological features, it did not impact GSK3 $\beta$ activation and accordingly phospho-tau levels were also unchanged (19). Phosphorylation of GSK3 $\beta$ can be attributed to enhanced insulin signalling and activation of IRS (37).

Different lifestyle modifications furthermore have an impact on markers of neuroinflammation and oxidative stress. Microglia and astrocytes reduce in number, reactivity, and size or complexity $(11,12,20,41)$, but also some favourable alternations in cytokine levels have been observed $(11,21,44)$. Some studies have shown that plaque-associated astrocytes decrease in number or complexity $(20,63)$, while others observe them as being more active around the plaques (13). The ameliorated neuroinflammation may be attributed to activation of the miR-129-5p (16) or inhibition of the JNK pathway and its down-stream targets $(11,44)$. Since JNK pathway is a down-stream target of receptor p75 (38), JNK pathway mediated reduction in neuroinflammation could be linked to changes in BDNF levels. A healthy lifestyle also maintains mitochondrial integrity and structure $(10,12)$, as well as mitochondrial function $(10,14,45$, 46). Regarding mitochondrial function, PGC- $1 \alpha$ not only 
indirectly promotes BDNF expression (32), but serves as a marker of mitochondrial biogenesis (12).

To incorporate this multitude of beneficial mechanisms that promote brain health, multidomain interventions targeting several risk factors and mechanisms are likely to be the most beneficial approach, given the heterogeneity and multifactorial nature of $\operatorname{AD}(70,71)$. Results from the pioneering multidomain lifestyle RCT - The Finnish Geriatric Intervention Study to Prevent Cognitive Impairment and Disability (FINGER) - supports the rationale for implementing multifactorial lifestyle changes, particularly in at-risk populations (72). Still, it should be mentioned that other multidomain lifestyle trials (e.g. preDIVA (Prevention of Dementia by Intensive Vascular Care) and MAPT (Multidomain Alzheimer Prevention Trial)) did not have measurable cognitive effects $(73,74)$.

Increased incidence of $\mathrm{AD}$ in global populations may be attributable to increased longevity, thus there is great need for promoting healthy ageing, especially in the context of brain health. The interventions reviewed here e.g. physical activity have also been shown to impact development of neuropathology, at least from studies in animal models. Further research is needed in earlier timepoints such as mid-life to evaluate the effect of lifestyle interventions on neurodegenerative brain changes in late-life. The diverse lifestyle-related interventions discussed in this paper have some clear benefits that are worth highlighting. First, they are all relatively easily available and do not necessarily require major financial input. Physical activity, a healthy diet, and cognitive challenges are further factors individuals may modify independently, in contrast to factors such as air pollution, traumatic head injury, and depression, which are all listed in Livingston et al.'s report as modifiable risk factors (4). Next, as encountered in the FINGER trial (72), the most common adverse event from such interventions is musculoskeletal pain. Thus, the disadvantages of healthy lifestyle changes are minor. Finally, an important aspect of such changes is the pervasive benefit of overall health. Not only was the CAIDE dementia risk score reduced (75), and cognitive functioning maintained or improved (72) in the FINGER trial, but additionally the risk of other chronic diseases and conditions related to lifestyle were diminished (76). Elucidating the mechanisms underpinning dementia prevention could yield precision medicine biomarkers and plausibly inform the discovery of novel therapeutic targets. Non-hypothesis-driven investigations to decipher the spectrum of mechanistic and cellular alterations in the brain as a result of lifestyle interventions could be a fruitful future avenue.

Funding: The sponsors had no role in the design and conduct of the study; in the collection, analysis, and interpretation of data; in the preparation of the manuscript; or in the review or approval of the manuscript.

Acknowledgments: This study was supported by the Swedish Research Council Center for Innovative Medicine (CIMED) at Flemingsberg Campus, Stiftelsen
Stockholms sjukhem, Sweden, Knut and Alice Wallenberg Foundation, Gun och Bertil Stohnes Stiftelse and Demensfonden.

Conflict of interest: The authors declare no disclosures.

Consent for publication: All authors have read the final version of the manuscript and have given their consent for publication.

Open Access: This article is distributed under the terms of the Creative Commons Attribution 4.0 International License (http:// creativecommons.org/ licenses/by/4.0/), which permits use, duplication, adaptation, distribution and reproduction in any medium or format, as long as you give appropriate credit to the original author(s) and the source, provide a link to the Creative Commons license and indicate if changes were made.

\section{References}

1. 2021 Alzheimer's disease facts and figures. Alzheimer's Dement 2021;17:327406. https: / / doi.org/10.1002/alz.12328

2. Ahmadi-Abhari S, Guzman-Castillo M, Bandosz P, et al. Temporal trend in dementia incidence since 2002 and projections for prevalence in England and Wales to 2040: Modelling study. BMJ 2017;358:j2856. https: / doi.org/10.1136/ bmj.j2856

3. Kivipelto M, Mangialasche F, Ngandu T. Lifestyle interventions to prevent cognitive impairment, dementia and Alzheimer disease. Nat Rev Neurol 2018;14:653-66. https: / / doi.org/10.1038/s41582-018-0070-3

4. Livingston G, Huntley J, Sommerlad A, et al. Dementia prevention, intervention, and care: 2020 report of the Lancet Commission. Lance 2020;396:413-46. https: / / doi.org/10.1016/S0140-6736(20)30367-6

5. World Health Organization. Risk reduction of cognitive decline and dementia: WHO guidelines. Geneva: CC BY-NC-SA 3.0 IGO; 2019. https: / /www.ncbi. nlm.nih.gov/books/NBK542796/

6. Yu JT, Xu W, Tan CC, et al. Evidence-based prevention of Alzheimer's disease: Systematic review and meta-analysis of 243 observational prospective studies and 153 randomised controlled trials. J Neurol Neurosurg Psychiatry 2020;91:1201-9. https: / / doi.org/10.1136/jnnp-2019-321913

7. Ozemek C, Tiwari S, Sabbahi A, Carbone S, Lavie CJ. Impact of therapeutic lifestyle changes in resistant hypertension. Prog Cardiovasc Dis 2020;63:4-9. https:/ / doi.org/10.1016/j.pcad.2019.11.012

8. Blüher M. Obesity: global epidemiology and pathogenesis. Nat Rev Endocrinol 2019;15:288-98. https: / / doi.org/10.1038/s41574-019-0176-8

9. Zheng Y, Ley SH, Hu FB. Global aetiology and epidemiology of type 2 diabetes mellitus and its complications. Nat Rev Endocrinol 2018;14:88-98. https: / / doi. org/10.1038/nrendo.2017.151

10. Pang R, Wang X, Pei F, et al. Regular Exercise Enhances Cognitive Function and Intracephalic GLUT Expression in Alzheimer's Disease Model Mice. J Alzheimer's Dis 2019;72:83-96. https:/ / doi.org/10.3233/JAD-190328

11. Liu Y, Chu JMT, Yan T, et al. Short-term resistance exercise inhibits neuroinflammation and attenuates neuropathological changes in $3 \times \mathrm{Tg}$ Alzheimer's disease mice. J Neuroinflammation 2020;17:4. https:/ / doi. org / 10.1186/s12974-019-1653-7

12. Kim D, Cho J, Kang H. Protective effect of exercise training against the progression of Alzheimer's disease in 3xTg-AD mice. Behav Brain Res 2019;374:112105. https: / doi.org/10.1016/j.bbr.2019.112105

13. Belaya I, Ivanova M, Sorvari A, et al. Astrocyte remodeling in the beneficial effects of long-term voluntary exercise in Alzheimer's disease. J Neuroinflammation 2020:17:271. https: / / doi.org/10.1186/s12974-020-01935-w

14. Park S-S, Park H-S, Kim C-J, et al. Physical exercise during exposure to $40-\mathrm{Hz}$ light flicker improves cognitive functions in the $3 \times \mathrm{Tg}$ mouse model of Alzheimer's disease. Alzheimers Res Ther 2020;12:62. https://doi. org/10.1186/S13195-020-00631-4

15. Choi DH, Kwon KC, Hwang DJ, et al. Treadmill Exercise Alleviates Brain Iron Dyshomeostasis Accelerating Neuronal Amyloid- $\beta$ Production, Neuronal Cell Death, and Cognitive Impairment in Transgenic Mice Model of Alzheimer's Disease. Mol Neurobiol 2021;58:3208-23. https: / / doi.org/10.1007/s12035-02102335-8

16. Li Z, Chen Q, Liu I, Du Y. Physical Exercise Ameliorates the Cognitive Function and Attenuates the Neuroinflammation of Alzheimer's Disease via miR-129-5p. Dement Geriatr Cogn Disord 2020;49:163-9. https:// doi org $/ 10.1159 / 000507285$

17. Zhang Y, Chao FL, Zhou CN, et al. Effects of exercise on capillaries in the white matter of transgenic AD mice. Oncotarget 2017;8:65860-75. https:/ / doi. org/10.18632/ oncotarget.19505

18. Lourenco M V., Frozza RL, de Freitas GB, et al. Exercise-linked FNDC5/irisin rescues synaptic plasticity and memory defects in Alzheimer's models. Nat Med 2019;25:165-75. https: / / doi.org/10.1038/s41591-018-0275-4

19. Kim TW, Park SS, Park JY, Park HS. Infusion of plasma from exercised mice ameliorates cognitive dysfunction by increasing hippocampal neuroplasticity and mitochondrial functions in 3xtg-ad mice. Int J Mol Sci 2020;21:3291. 
https: / / doi.org/10.3390/ijms21093291

20. Zhang J, Guo Y, Wang Y, et al. Long-term treadmill exercise attenuates $\mathrm{A} \beta$ burdens and astrocyte activation in APP/PS1 mouse model of Alzheimer's disease. Neurosci Lett 2018;666:70-7. https://doi.org/10.1016/j. neulet.2017.12.025

21. Do K, Laing BT, Landry $\mathrm{T}$, et al. The effects of exercise on hypothalamic neurodegeneration of Alzheimer's disease mouse model. PLoS One 2018;13:e0190205. https:/ / doi.org/10.1371/journal.pone.0190205

22. Bartzokis G, Cummings JL, Sultzer D, et al. White matter structural integrity in healthy aging adults and patients with Alzheimer disease: A magnetic resonance imaging study. Arch Neurol 2003;60:393-8. https://doi. org/10.1001/archneur.60.3.393

23. Zhou CN, Chao FL, Zhang Y, et al. Sex differences in the white matter and myelinated fibers of APP/PS1 mice and the effects of running exercise on the sex differences of AD mice. Front Aging Neurosci 2018;10:243. https://doi. org $/ 10.3389 /$ fnagi.2018.00243

24. Bao C, He C, Shu B, et al. Aerobic exercise training decreases cognitive impairment caused by demyelination by regulating ROCK signaling pathway in aging mice. Brain Res Bull 2021;168:52-62. https://doi.org/10.1016/j. brainresbull.2020.12.010

25. Sack M, Lenz JN, Jakovcevski M, et al. Early effects of a high-caloric diet and physical exercise on brain volumetry and behavior: a combined MRI and histology study in mice. Brain Imaging Behav 2017;11:1385-96. https:/ / doi. org /10.1007/s11682-016-9638-y

26. McKenzie IA, Ohayon D, Li H, et al. Motor skill learning requires active central myelination. Science (80- ) 2014;346:318-22. https:// doi.org/10.1126/ science. 1254960

27. Fujita $Y$, Yamashita T. Axon growth inhibition by RhoA/ROCK in the central nervous system. Front Neurosci 2014;8:338. https://doi.org/10.3389/ fnins.2014.00338

28. Baer AS, Syed YA, Kang SU, et al. Myelin-mediated inhibition of oligodendrocyte precursor differentiation can be overcome by pharmacological modulation of Fyn-RhoA and protein kinase $\mathrm{C}$ signalling. Brain 2009;132:465-81. https:/ / doi.org/10.1093/brain/awn334

29. Bashiri H, Enayati M, Bashiri A, Salari AA. Swimming exercise improves cognitive and behavioral disorders in male NMRI mice with sporadic Alzheimer-like disease. Physiol Behav 2020;223:113003. https:// doi. org/10.1016/j.physbeh.2020.113003

30. Horowitz AM, Fan X, Bieri G, et al. Blood factors transfer beneficial effects of exercise on neurogenesis and cognition to the aged brain. Science (80- ) 2020;369:167-73. https: / / doi.org/10.1126/ science.aaw2622

31. Um HS, Kang EB, Leem YH, et al. Exercise training acts as a therapeutic strategy for reduction of the pathogenic phenotypes for Alzheimer's disease in an NSE/ APPsw-transgenic model. Int J Mol Med 2008;22:529-39. https:/ / doi. org/10.3892/IJMM_00000052

32. Wrann CD, White JP, Salogiannnis J, et al. Exercise induces hippocampal BDNF through a PGC-1 $\alpha$ /FNDC5 pathway. Cell Metab 2013;18:649-59. https:/ / doi.org/10.1016/j.cmet.2013.09.008

33. Teng HK, Teng KK, Lee R, et al. ProBDNF induces neuronal apoptosis via activation of a receptor complex of p75NTR and sortilin. J Neurosci 2005;25:5455-63. https: / / doi.org/10.1523/JNEUROSCI.5123-04.2005

34. Wang M, Xie $Y$, Qin D. Proteolytic cleavage of proBDNF to mBDNF in neuropsychiatric and neurodegenerative diseases. Brain Res Bull 2021;166:17284. https: / / doi.org/10.1016/j.brainresbull.2020.11.005

35. Reuland CJ, Church FC. Synergy between plasminogen activator inhibitor-1, $\alpha$-synuclein, and neuroinflammation in Parkinson's disease. Med Hypotheses 2020;138:109602. https:/ / doi.org/10.1016/j.mehy.2020.109602

36. Ding Q, Ying Z, Gómez-Pinilla F. Exercise influences hippocampal plasticity by modulating brain-derived neurotrophic factor processing. Neuroscience 2011;192:773-80. https: / / doi.org/10.1016/j.neuroscience.2011.06.032

37. Park HS, Park SS, Kim CJ, Kim TW, Kim TW. Exercise alleviates cognitive functions by enhancing hippocampal insulin signaling and neuroplasticity in high-fat diet-induced obesity. Nutrients 2019;11:1603. https:// doi. org $/ 10.3390 /$ nu11071603

38. Houlton J, Abumaria N, Hinkley SFR, Clarkson AN. Therapeutic potential of neurotrophins for repair after brain injury: A helping hand from biomaterials. Front Genet 2019;10. https:/ / doi.org/10.3389/ fnins.2019.00790

39. Levin F, Ferreira D, Lange C, et al. Data-driven FDG-PET subtypes of Alzheimer's disease-related neurodegeneration. Alzheimer's Res Ther 2021;13:49. https: / / doi.org/10.1186/s13195-021-00785-9

40. Ismail M-A-M, Mateos L, Maioli S, et al. 27-Hydroxycholesterol impairs neuronal glucose uptake through an IRAP/GLUT4 system dysregulation. J Exp Med 2017;214:699-717. https:/ / doi.org/10.1084/jem.20160534

41. Lauretti E, Iuliano L, Praticò D. Extra-virgin olive oil ameliorates cognition and neuropathology of the 3xTg mice: role of autophagy. Ann Clin Transl Neurol 2017;4:564-74. https: / / doi.org/10.1002/acn3.431

42. Qosa H, Mohamed LA, Batarseh YS, et al. Extra-virgin olive oil attenuates amyloid- $\beta$ and tau pathologies in the brains of TgSwDI mice. J Nutr Biochem
2015;26:1479-90. https:/ / doi.org/10.1016/j.jnutbio.2015.07.022

43. Pandey KB, Rizvi SI. Plant polyphenols as dietary antioxidants in human health and disease. Oxid Med Cell Longev 2009;2:270-8. https://doi. org/10.4161/oxim.2.5.9498

44. Peng Y, Hou C, Yang Z, et al. Hydroxytyrosol mildly improve cognitive function independent of APP processing in APP/PS1 mice. Mol Nutr Food Res 2016;60:2331-42. https:/ / doi.org/10.1002/mnfr.201600332

45. Grewal R, Reutzel M, Dilberger B, et al. Purified oleocanthal and ligstroside protect against mitochondrial dysfunction in models of early Alzheimer's disease and brain ageing. Exp Neurol 2020;328:113248. https:// doi. org / 10.1016/j.expneurol.2020.113248

46. Reutzel M, Grewal R, Silaidos C, et al. Effects of long-term treatment with a blend of highly purified olive secoiridoids on cognition and brain ATP levels in aged NMRI mice. Oxid Med Cell Longev 2018;2018:4070935. https://doi. org / 10.1155/2018/4070935

47. Grossi C, Rigacci S, Ambrosini S, et al. The Polyphenol Oleuropein Aglycone Protects TgCRND8 Mice against Aß Plaque Pathology. PLoS One 2013;8:e71702. https:// doi.org/10.1371/journal.pone.0071702

48. Luccarini I, Grossi C, Rigacci S, et al. Oleuropein aglycone protects against pyroglutamylated-3 amyloid- $\beta$ toxicity: Biochemical, epigenetic and functional correlates. Neurobiol Aging 2015;36:648-63. https:// doi.org/10.1016/j. neurobiolaging.2014.08.029

49. Van Wijk N, Broersen LM, De Wilde MC, et al. Targeting synaptic dysfunction in Alzheimer's disease by administering a specific nutrient combination. J Alzheimer's Dis 2014;38:459-79. https:/ / doi.org/10.3233/JAD-130998

50. Koivisto H, Grimm MO, Rothhaar TL, et al. Special lipid-based diets alleviate cognitive deficits in the APPswe/PS1dE9 transgenic mouse model of Alzheimer's disease independent of brain amyloid deposition. J Nutr Biochem 2014;25:157-69. https:/ / doi.org/10.1016/j.jnutbio.2013.09.015

51. Wiesmann M, Jansen D, Zerbi V, et al. Improved spatial learning strategy and memory in aged Alzheimer AßPPswe/PS1dE9 mice on a multi-nutrient diet. J Alzheimer's Dis 2013;37:233-45. https:/ / doi.org/10.3233/JAD-130179

52. Jansen D, Zerbi V, Arnoldussen IAC, et al. Effects of Specific Multi-Nutrient Enriched Diets on Cerebral Metabolism, Cognition and Neuropathology in AßPPswe-PS1dE9 Mice. PLoS One 2013;8:e75393. https:// doi.org/10.1371/ journal.pone.0075393

53. Jansen D, Zerbi V, Janssen CIF, et al. Impact of a multi-nutrient diet on cognition, brain metabolism, hemodynamics, and plasticity in apoE4 carrier and apoE knockout mice. Brain Struct Funct 2014;219:1841-68. https://doi. org / 10.1007/ s00429-013-0606-7

54. Broersen LM, Kuipers AAM, Balvers M, et al. A Specific multi-nutrient diet reduces alzheimer-like pathology in young adult aßPPswe/PS1dE9 Mice. J Alzheimer's Dis 2013;33:177-90. https:/ / doi.org/10.3233/JAD-2012-112039

55. Rai SP, Krohn M, Pahnke J. Early Cognitive Training Rescues Remote Spatia Memory but Reduces Cognitive Flexibility in Alzheimer's Disease Mice. J Alzheimer's Dis 2020;75:1105-34. https:// doi.org/10.3233/JAD-200161

56. Jiang X, Chai GS, Wang ZH, et al. Spatial training preserves associative memory capacity with augmentation of dendrite ramification and spine generation in Tg2576 mice. Sci Rep 2015;5:9488. https:// doi.org/10.1038/ srep09488

57. Billings LM, Green KN, McGaugh JL, LaFerla FM. Learning decreases $A \beta^{*} 56$ and tau pathology and ameliorates behavioral decline in 3xTg-AD mice. J Neurosci 2007;27:751-61. https:/ / doi.org/10.1523/JNEUROSCI.4800-06.2007

58. Daumas S, Sandin J, Chen KS, et al. Faster forgetting contributes to impaired spatial memory in the PDAPP mouse: Deficit in memory retrieval associated with increased sensitivity to interference? Learn Mem 2008;15:625-32. https:/ / doi.org/10.1101/lm.990208

59. Harrison FE, Hosseini AH, McDonald MP. Endogenous anxiety and stress responses in water maze and Barnes maze spatial memory tasks. Behav Brain Res 2009;198:247-51. https:/ / doi.org/10.1016/j.bbr.2008.10.015

60. Nithianantharajah J, Hannan AJ. Enriched environments, experiencedependent plasticity and disorders of the nervous system. Nat Rev Neurosci 2006;7:697-709. https: / / doi.org/10.1038/nrn1970

61. Verret L, Krezymon A, Halley H, et al. Transient enriched housing before amyloidosis onset sustains cognitive improvement in Tg2576 mice. Neurobio Aging 2013;34:211-25. https:/ / doi.org/10.1016/j.neurobiolaging.2012.05.013

62. Polito L, Chierchia A, Tunesi M, et al. Environmental enrichment lessen cognitive decline in APP23 mice without affecting brain sirtuin expression. J Alzheimer's Dis 2014;42:851-64. https:/ / doi.org/10.3233/JAD-131430

63. Beauquis J, Pavía P, Pomilio C, et al. Environmental enrichment prevents astroglial pathological changes in the hippocampus of APP transgenic mice, model of Alzheimer's disease. Exp Neurol 2013;239:28-37. https://doi. org/10.1016/j.expneurol.2012.09.009

64. Wolf SA, Kronenberg G, Lehmann K, et al. Cognitive and Physical Activity Differently Modulate Disease Progression in the Amyloid Precursor Protein (APP)-23 Model of Alzheimer's Disease. Biol Psychiatry 2006;60:1314-23. https:// doi.org/10.1016/j.biopsych.2006.04.004

65. Andrieu S, Coley N, Lovestone S, Aisen PS, Vellas B. Prevention of sporadic 
Alzheimer's disease: Lessons learned from clinical trials and future directions. Lancet Neurol 2015;14:926-44. https: / / doi.org/10.1016/S1474-4422(15)00153-2

66. Hodge RD, Bakken TE, Miller JA, et al. Conserved cell types with divergen features in human versus mouse cortex. Nature 2019;573:61-8. https: / doi. org/10.1038/s41586-019-1506-7

67. Scearce-Levie K, Sanchez PE, Lewcock JW. Leveraging preclinical models for the development of Alzheimer disease therapeutics. Nat Rev Drug Discov 2020;19:447-62. https: / / doi.org/10.1038/s41573-020-0065-9

68. Gupta V, Chitranshi N, You Y, et al. Brain derived neurotrophic factor is involved in the regulation of glycogen synthase kinase $3 \beta$ (GSK3 $\beta$ ) signalling. Biochem Biophys Res Commun 2014;454:381-6. https://doi.org/10.1016/j. bbrc.2014.10.087

69. Llorens-Martín M, Jurado J, Hernández F, Ávila J. GSK-3 $\beta$, a pivotal kinase in Alzheimer disease. Front Mol Neurosci 2014;7:46. https:/ / doi.org/10.3389/ fnmol.2014.00046

70. Boyle PA, Yu L, Leurgans SE, et al. Attributable risk of Alzheimer's dementia attributed to age-related neuropathologies. Ann Neurol 2019;85:114-24. https: / / doi.org/10.1002/ana.25380

71. Tijms BM, Gobom J, Reus L, et al. Pathophysiological subtypes of Alzheimer's disease based on cerebrospinal fluid proteomics. Brain 2020;143:3776-92. https: / / doi.org/10.1093/brain/awaa325

72. Ngandu T, Lehtisalo J, Solomon A, et al. A 2 year multidomain intervention of diet, exercise, cognitive training, and vascular risk monitoring versus contro to prevent cognitive decline in at-risk elderly people (FINGER): A randomised controlled trial. Lancet 2015;385:2255-63. https: / / doi.org/10.1016/S01406736(15)60461-5
73. Andrieu S, Guyonnet S, Coley N, et al. Effect of long-term omega 3 polyunsaturated fatty acid supplementation with or without multidomain intervention on cognitive function in elderly adults with memory complaints (MAPT): a randomised, placebo-controlled trial. Lancet Neurol 2017;16:377-89. https: / / doi.org/10.1016/S1474-4422(17)30040-6

74. van Charante EPM, Richard E, Eurelings LS, et al. Effectiveness of a 6-year multidomain vascular care intervention to prevent dementia (preDIVA): a cluster-randomised controlled trial. Lancet 2016;388:797-805. https: / / doi. org/10.1016/S0140-6736(16)30950-3

75. Solomon A, Handels R, Wimo A, et al. Effect of a multidomain lifestyle intervention on estimated dementia risk. J Alzheimer's Dis 2021;82:1461-6. https: / / doi.org/10.3233/JAD-210331

76. Marengoni A, Rizzuto D, Fratiglioni L, et al. The Effect of a 2-Year Intervention Consisting of Diet, Physical Exercise, Cognitive Training, and Monitoring of Vascular Risk on Chronic Morbidity-the FINGER Randomized Controlled Trial. J Am Med Dir Assoc 2018;19:355-360.e1. https:// doi.org/10.1016/j. jamda.2017.09.020

How to cite this article: V. Alanko, C. Udeh-Momoh, M. Kivipelto, et al. Mechanisms Underlying Non-Pharmacological Dementia Prevention Strategies: A Translational Perspective. J Prev Alz Dis 2022;1(9):3-11, http:/ / dx.doi.org/10.14283/jpad.2022.9 